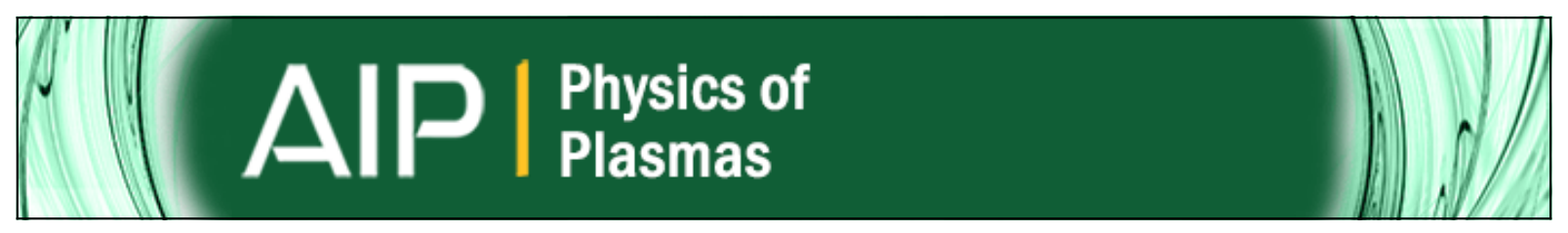

\title{
Three dimensional character of whistler turbulence
}

Gurudas Ganguli, Leonid Rudakov, Wayne Scales, Joseph Wang, and Manish Mithaiwala

Citation: Physics of Plasmas (1994-present) 17, 052310 (2010); doi: 10.1063/1.3420245

View online: http://dx.doi.org/10.1063/1.3420245

View Table of Contents: http://scitation.aip.org/content/aip/journal/pop/17/5?ver=pdfcov

Published by the AIP Publishing

\section{AlP Re-register for Table of Content Alerts}




\title{
Three dimensional character of whistler turbulence
}

\author{
Gurudas Ganguli, ${ }^{1, a)}$ Leonid Rudakov, ${ }^{2}$ Wayne Scales, ${ }^{3}$ Joseph Wang, ${ }^{4}$ \\ and Manish Mithaiwala ${ }^{1}$ \\ ${ }^{1}$ Plasma Physics Division, Naval Research Laboratory, Washington, DC 20375-5346, USA \\ ${ }^{2}$ Icarus Research Inc., P.O. Box 30780, Bethesda, Maryland 20824-0780, USA \\ ${ }^{3}$ Bradley Department of Electrical and Computer Engineering, Virginia Tech, \\ Blacksburg, Virginia 24061-0111, USA \\ ${ }^{4}$ Astronautical Engineering Division, University of Southern California, \\ Los Angles, California 90089-1192, USA
}

(Received 31 December 2009; accepted 5 April 2010; published online 18 May 2010)

\begin{abstract}
It is shown that the dominant nonlinear effect makes the evolution of whistler turbulence essentially three dimensional in character. Induced nonlinear scattering due to slow density perturbation resulting from ponderomotive force triggers energy flux toward lower frequency. Anisotropic wave vector spectrum is generated by large angle scatterings from thermal plasma particles, in which the wave propagation angle is substantially altered but the frequency spectrum changes a little. As a consequence, the wave vector spectrum does not indicate the trajectory of the energy flux. There can be conversion of quasielectrostatic waves into electromagnetic waves with large group velocity, enabling convection of energy away from the region. We use a two-dimensional electromagnetic particle-in-cell model with the ambient magnetic field out of the simulation plane to generate the essential three-dimensional nonlinear effects. (C) 2010 American Institute of Physics.
\end{abstract}

[doi:10.1063/1.3420245]

\section{INTRODUCTION}

Turbulence facilitates transport and distribution of energy and momentum in a collisionless plasma medium. Therefore, evolution of electromagnetic turbulence is important to both space and laboratory plasmas and has been a topic of intense research for decades. Electromagnetic whistler turbulence in the intermediate frequency range, $\Omega_{i} \ll \omega \ll \Omega_{e}$, where $\Omega_{i(e)}$ are ion (electron) gyrofrequency, continues to be of interest to ionospheric, ${ }^{1,2}$ magnetospheric, and solar wind plasmas ${ }^{3-5}$ as well as to laboratory plasmas. ${ }^{6,7}$ The purpose of this article is to show that the dominant nonlinear (NL) effect makes whistler turbulence in a low $\beta$ plasma, similar to that found in the near-earth space environment, a three-dimensional (3D) phenomenon in which the evolution of the turbulence is characterized by induced NL scattering. NL scattering arises due to a slow density perturbation resulting from the ponderomotive force acting along the ambient magnetic field lines. Induced scattering can result in a large change in propagation angle of the waves and consequently in conversion of quasielectrostatic waves into electromagnetic waves, and vice versa. Since group velocities of quasielectrostatic and electromagnetic waves are very different, this conversion can lead to a significantly different final NL plasma state.

In Sec. II we first discuss the linear electromagnetic dispersion relation of the waves in the intermediate frequency range. Then we discuss the NL properties of the turbulence due to these waves and provide the NL growth rate of the induced scattering. In Sec. III we describe our electromagnetic particle-in-cell (PIC) simulation and employ it to

\footnotetext{
${ }^{a)}$ Electronic mail: gurudas.ganguli@nrl.navy.mil.
}

examine the NL scattering properties of the intermediate frequency waves. Finally in Sec IV we provide our conclusions.

\section{THEORY}

In the intermediate frequency range there is a relative drift between the effectively unmagnetized ions and the magnetized electrons resulting in a total current, $\vec{j}$, which includes the displacement current and can be expressed as

$$
\begin{aligned}
\vec{j}= & -\frac{c}{4 \pi} \nabla^{2} \vec{A} \\
= & -e n_{0}\left[\frac{c \vec{E} \times \vec{b}}{B_{0}}-i \frac{e E_{\|} \vec{b}_{0}}{m_{e} \omega}+\frac{i \omega}{\Omega_{e}}\left(1-\frac{\omega_{\mathrm{LH}}^{2}}{\omega^{2}}\right)\right. \\
& \left.\times \frac{c \vec{E}_{\perp}}{B_{0}}+\frac{i \omega}{\Omega_{e}} \frac{\Omega_{e}^{2}}{\omega_{p e}^{2}} \frac{c \vec{E}}{B_{0}}\right] .
\end{aligned}
$$

For simplicity we consider a dense plasma, $\omega_{p e}^{2} \gg \Omega_{e}^{2}$, and ignore the last term in Eq. (1). $\vec{E}$ is the wave electric fluctuation, $\vec{A}$ is the vector potential, $B_{0}$ is the background magnetic field along the $z$-axis, $\vec{b}=\vec{B}_{0} /\left|B_{0}\right|, \omega_{\mathrm{LH}}=\sqrt{\Omega_{e} \Omega_{i}}$ is the lower hybrid (LH) frequency, and $\omega_{p e}$ is the electron plasma frequency. Maxwell equations along with the Coulomb gauge yields for plane waves $\sim \exp \left(-i \omega t+i k_{x} x+i k_{z} z\right)$,

$$
E_{x}=-i k_{x} \varphi \frac{\bar{k}^{2}}{\bar{k}_{\perp}^{2}}, \quad E_{y}=E_{x} \frac{i \omega}{\Omega_{e} \bar{k}^{2}}, \quad E_{z}=-i k_{z} \varphi \frac{\bar{k}^{2}}{\left(1+\bar{k}^{2}\right)},
$$

where $\varphi$ is the electrostatic potential. $E_{y}$ is of purely electromagnetic origin while $E_{x}$ and $E_{z}$ can become electrostatic for 
short wavelengths and $k_{\perp} \gg k_{\|}$. Wave vector is normalized by the electron skin depth, i.e., $\bar{k}=k c / \omega_{\text {pe }}, \bar{k}^{2}=\vec{k}_{\|}^{2}+\vec{k}_{\perp}^{2}, k_{\perp}=k_{x}$, and the subscripts "||" and " $\perp$ " are with respect to $B_{0}$. Setting $\nabla \cdot \bar{j}=0$ and using Eq. (2) to eliminate the electric fields we obtain the dispersion relation for the intermediate frequency range waves in a cold plasma,

$$
\omega^{2}=\left(\frac{\vec{k}_{\|}^{2}}{\left(1+\vec{k}_{\perp}^{2}\right)}+\frac{\omega_{\mathrm{LH}}^{2}}{\Omega_{e}^{2}}\right) \frac{\vec{k}^{2} \Omega_{e}^{2}}{1+\vec{k}^{2}} .
$$

For short perpendicular wavelengths, $\bar{k}_{\perp} \gg 1$, and small propagation angles, $k_{\|} / k_{\perp} \ll \omega_{\mathrm{LH}} / \Omega_{e}$, Eq. (3) reduces to $\omega^{2}=\omega_{\mathrm{LH}}^{2}$, the dispersion relation for the LH waves. In the LH limit $E_{y} \rightarrow 0$ and $E_{x, \|} \rightarrow-\nabla_{x, \|} \varphi$, implying that the waves are electrostatic in character. For long perpendicular wavelengths, $\bar{k}_{\perp}<1$, and $\bar{k}_{\|}>\omega_{\mathrm{LH}} / \Omega_{e}$, Eq. (3) reduces to $\omega^{2}=\bar{k}_{\|}^{2} \bar{k}^{2} \Omega_{e}^{2}$, the dispersion relation for the whistler (W) waves. For long perpendicular wavelengths, $\bar{k}_{\perp}<1$, and $\bar{k}_{\|}<\omega_{\mathrm{LH}} / \Omega_{e}$, Eq. (3) reduces to the dispersion relation for the magnetosonic (MS) waves, $\omega^{2}=k^{2} V_{A}^{2}$, where $V_{A}$ $=B_{0} / \sqrt{4 \pi n_{i} m_{i}}$ is the Alfven speed. Clearly, the LH, MS, and $\mathrm{W}$ waves belong to the same branch but at different propagation angles and are formally electromagnetic. As we shall see this fact is quite important in understanding the nature of turbulence in the intermediate frequency range.

In a nonuniform plasma the intermediate frequency mode structure is significantly altered. To clarify this we replace $n_{0}$ by $n_{0}+\delta n$ in Eq. (1), where $\delta n$ represents the inhomogeneity that may be external or self-consistently generated by the ponderomotive force. With this substitution and $\nabla \cdot \bar{j}=0$ Eq. (1) gives

$$
\begin{aligned}
& n_{0} \frac{\omega}{\Omega_{e}}\left(\frac{1+\vec{k}^{2}}{\vec{k}^{2}}-\frac{\omega_{\mathrm{LH}}^{2}}{\omega^{2}}-\frac{\Omega_{e}^{2}}{\omega^{2}} \frac{\vec{k}_{\|}^{2}}{1+\vec{k}_{\perp}^{2}}\right) \frac{c \vec{\nabla}_{\perp} \cdot \vec{E}_{\perp}}{B_{0}} \\
& +\frac{i c}{B_{0}}(\vec{E} \times \vec{\nabla} \delta n) \cdot \vec{b}_{0}=0 .
\end{aligned}
$$

Solving Eq. (4) the new dispersion relation is

$$
\begin{aligned}
\frac{\omega}{\Omega_{e}}= & -\frac{(\vec{k} \times \vec{\nabla} \delta n) \cdot \vec{b}}{2 n_{0} k^{2}} \frac{\vec{k}^{2}}{1+\bar{k}^{2}} \\
& \pm\left\{\left[\frac{(\vec{k} \times \vec{\nabla} \delta n) \cdot \vec{b}}{2 n_{0} k^{2}} \frac{\bar{k}^{2}}{1+\bar{k}^{2}}\right]^{2}+\frac{\omega_{\mathrm{LH}}^{2} \bar{k}^{2}}{\Omega_{e}^{2}\left(1+\bar{k}^{2}\right)}\right. \\
& \left.+\frac{\vec{k}_{\|}^{2} \bar{k}^{2}}{\left(1+\vec{k}_{\perp}^{2}\right)\left(1+\bar{k}^{2}\right)}\right\}^{1 / 2} .
\end{aligned}
$$

If $\delta n / n>\max \left[\left(m_{e} / m_{i}\right)^{1 / 2},\left(k_{\|} / k_{\perp}\right)\right]$ then Eq. (5) yields a drift mode-like oscillation

$$
\frac{\omega}{\Omega_{e}}=-\frac{(\vec{k} \times \vec{\nabla} \delta n) \cdot \vec{b}}{n_{0} k^{2}} \frac{\bar{k}^{2}}{1+\bar{k}^{2}} .
$$

When $\delta n$ is self-consistently generated by the ponderomotive force along the magnetic field, the new term proportional to $\delta n$ in Eq. (4) becomes 3D and is the source of the so-called vector nonlinearity, ${ }^{8}$ which can significantly affect the turbulence dynamics by making it a 3D phenomenon and inducing strong NL scatterings. This vector nonlinearity related $\delta n$ dependence on the real frequency [Eq. (5)] is much stronger than the scalar nonlinearity related $\delta n$ dependence that would appear through the plasma frequency, as in the NL evolution of Langmuir waves.'

We calculate this second order electron density perturbation $\delta n_{e}^{(2)}$ by using the Vlasov equation in drift approximation and assuming a Maxwellian distribution. Ions are treated as a fluid and their motion under second order ambipolar electric field maintains quasineutrality. This leads to

$$
\begin{aligned}
\frac{\delta n_{e}^{(2)}}{n_{0}}= & \left(i \frac{\left(\vec{k}_{2}-\vec{k}_{1}\right)^{2}}{\left(k_{2 z}-k_{1 z}\right) \Omega_{e}}\left(\frac{k_{2 z} \vec{k}_{2}^{2}}{\omega_{k 2}\left(1+\vec{k}_{2}^{2}\right)}-\frac{k_{1 z} \vec{k}_{1}^{2}}{\omega_{k 1}\left(1+\vec{k}_{1}^{2}\right)}\right)\right. \\
& \left.\times \frac{\left(\vec{E}_{1}^{*} \times \vec{E}_{2}\right) \cdot \vec{b}}{4 \pi n_{0} m_{e}}\right) \frac{\varepsilon_{k 1-k 2}^{i} \varepsilon_{k 1-k 2}^{e}}{\varepsilon_{k 1-k 2}^{e}+\varepsilon_{k 1-k 2}^{i}},
\end{aligned}
$$

where $\varepsilon_{k 1-k 2}^{i}, \varepsilon_{k 1-k 2}^{e}$ are the low frequency $\left(\omega_{k 1}-\omega_{k 2} \ll \omega_{k 1}\right)$ ion and electron susceptibilities. Superscript “*” implies complex conjugate, and subscripts "1" and "2" denote mother and daughter waves respectively. Substituting Eq. (7) in Eq. (4) we obtain the rate of NL scattering $\left(\gamma_{\mathrm{NL}}\right)$.

For a narrow band wave spectrum $\left(\delta \omega \ll \gamma_{\mathrm{NL}}\right)$ the NL rate is given by

$$
\begin{aligned}
\gamma_{\mathrm{NL}}^{2} \sim & \frac{1}{\omega_{k 2}} \frac{\vec{k}_{2}^{2}}{1+\vec{k}_{2}^{2}} \frac{\left|E_{k 1}\right|^{2}}{4 \pi n_{0} m_{e}} \frac{\left(\vec{k}_{1} \times \vec{k}_{2}\right)_{\|}^{2}}{k_{\perp 1}^{2} k_{\perp 2}^{2}} \frac{\left(\vec{k}_{2}-\vec{k}_{1}\right)^{2} \vec{k}_{1}^{2}}{\left(1+\vec{k}_{1}^{2}\right)} \\
& \times\left|\frac{\varepsilon_{k 1-k 2}^{i} \varepsilon_{k 1-k 2}^{e}}{\partial\left(\varepsilon_{k 1-k 2}^{i}+\varepsilon_{k 1-k 2}^{e}\right) / \partial \omega}\right|,
\end{aligned}
$$

which represents parametric decay of the intermediate frequency waves. However, in a broadband turbulence with incoherent wave spectrum $\left(\delta \omega \gg \gamma_{\mathrm{NL}}\right)$ the growth rate is averaged over the spectrum of wave vectors. This leads to the NL rate applicable to a broadband turbulence to be

$$
\begin{aligned}
\gamma_{\mathrm{NL}} \sim & \frac{1}{\omega_{k 2}} \frac{\vec{k}_{2}^{2}}{1+\vec{k}_{2}^{2}} \sum_{k 1} \frac{\left|E_{k 1}\right|^{2}}{4 \pi n_{0} m_{e}} \frac{\left(\vec{k}_{1} \times \vec{k}_{2}\right)_{\|}^{2}}{k_{\perp 1}^{2} k_{\perp 2}^{2}} \frac{\left(\vec{k}_{2}-\vec{k}_{1}\right)^{2} \vec{k}_{1}^{2}}{\left(1+\vec{k}_{1}^{2}\right)} \\
& \times\left\{\left|\frac{\varepsilon_{k 1-k 2}^{i} \varepsilon_{k 1-k 2}^{e}}{\partial\left(\varepsilon_{k 1-k 2}^{i}+\varepsilon_{k 1-k 2}^{e}\right) / \partial \omega}\right| \pi \delta\left(\omega_{k 1}-\omega_{k 2}-\omega_{k 1-k 2}\right)\right. \\
& \left.+\frac{\operatorname{Im} \varepsilon_{k 1-k 2}^{i}\left|\varepsilon_{k 1-k 2}^{e}\right|^{2}+\operatorname{Im} \varepsilon_{k 1-k 2}^{e}\left|\varepsilon_{k 1-k 2}^{i}\right|^{2}}{\left|\varepsilon_{k 1-k 2}^{e}+\varepsilon_{k 1-k 2}^{i}\right|^{2}}\right\}
\end{aligned}
$$

Equation (9) is the induced NL scattering rate for both wavewave and wave-particle scattering. The first term gives the scattering rate of the mother wave into a daughter wave through radiation of a lower frequency $\left(\omega_{k 1-k 2}\right)$ wave. In such wave-wave scattering event energy and momentum are conserved within the waves, i.e., $\omega_{k 1}=\omega_{k 2}+\omega_{k 3}, \vec{k}_{1}=\vec{k}_{2}+\vec{k}_{3}$, and the plasma serves as an oscillating medium with no exchange of momentum and energy with the waves. The entropy of the waves $S_{w}=\ln \Sigma_{k} N_{k}$, where $N_{k}=W_{k} / \omega_{k}$ is the plasmon number density, increases but their energy $\Sigma_{k} W_{k}$ is not dissipated. ${ }^{10}$ The second term in Eq. (9) gives the scattering rate of the mother into a daughter wave through resonance 
with plasma particles. In each such wave-particle scattering event the wave frequency decreases and the balance of wave energy is dissipated as plasma heating. As a result the plasma entropy increases and the process is irreversible. In addition, the number of plasmons do not change and the momentum conservation involves the principal momentum of magnetized electrons $\left(m_{e} \overrightarrow{\mathrm{v}}_{\perp e}+e \vec{A} / c\right)$, which includes the external magnetic field. The external magnetic field can absorb wave momentum and facilitate large change in scattered wave vectors.

Now consider the evolution of 3D broadband LH/W turbulence in an isothermal $\left(T_{i}=T_{e}\right)$, low $\beta$ plasma which is representative of the near-earth space environment. In this situation NL scattering by plasma particles dominates because there is no ion sound wave and hence the decay through radiation of ion sound wave is not possible. The NL scattering results from the Landau resonance of beat waves with magnetized electrons, i.e., $\left(\omega_{k 1}-\omega_{k 2}\right)=\left(k_{1 \|}-k_{2 \|}\right) \mathrm{v}_{\| e}$. In principle, resonance of the beat waves with ions is also possible. However for $\left|\left(k_{1 \|}-k_{2 \|}\right)\right| \mathrm{v}_{t i}<\Omega_{i}$, which is likely in a low $\beta$ plasma, the ion motion in the presence of high frequency $\left(\omega_{k 1}-\omega_{k 2} \gg \Omega_{i}\right)$ waves can be treated as unmagnetized but with no ion Landau damping. ${ }^{11}$ This makes the NL scattering through thermal electrons to be the dominant scattering mechanism in a low $\beta$ isothermal plasma.

Parametric decay of LH waves was extensively studied in fusion plasmas in connection to plasma heating in tokamaks. ${ }^{12}$ A comprehensive analysis of this decay process for the more general case was given by Tripathi et al. ${ }^{13}$ Hasegawa and Chen ${ }^{14}$ investigated the NL scattering of the LH waves by thermal electrons in a broadband turbulence using an electrostatic formalism. For $\mathrm{LH} \rightarrow \mathrm{LH}$ scattering rate in the electrostatic limit Eq. (9) simplifies to

$$
\gamma_{\mathrm{NL}}=\omega_{\mathrm{LH}} \frac{m_{i}}{m_{e}} \sum_{k 1} \frac{\left(\vec{k}_{1} \times \vec{k}_{2}\right)_{\|}^{2}}{k_{1 \perp}^{2} k_{2 \perp}^{2}} \pi \zeta \operatorname{Im} Z(\zeta) \frac{W_{k 1}}{n_{0} T_{e}},
$$

where $W_{k} \equiv\left(\omega_{p e}^{2} / \Omega_{e}^{2}\right)\left(\left|\vec{E}_{k}\right|^{2} / 8 \pi\right)$ is the LH wave energy density, which is primarily due to energy of the electron $E \times B$ drift, $\zeta=\left(\omega_{k 1}-\omega_{k 2}\right) /\left(k_{1 \|}-k_{2 \|}\right) \mathrm{v}_{t e}, \mathrm{v}_{t e}=\sqrt{2 T_{e} / m_{e}}$ is the electron thermal speed. In Eq. (10) subsonic condition $\left(\omega_{k 1}-\omega_{k 2}\right)$ $<\left(\vec{k}_{1}-\vec{k}_{2}\right) c_{s}$ is assumed where $c_{s}=\sqrt{T_{e} / m_{i}}$.

The LH wave frequency obtained from Eq. (3) in the electrostatic limit $\left(\bar{k}_{\perp} \gg 1\right.$ and $\left.k_{\|} / k_{\perp} \ll 1\right)$ becomes $\omega=\omega_{\mathrm{LH}}$ $\times\left(1+k_{\|}^{2} m_{i} / k_{\perp}^{2} m_{e}\right)^{1 / 2}$, and has a minimum at $\omega=\omega_{\mathrm{LH}}$. Since $\omega_{k 2}<\omega_{k 1}$, repeated scattering decreases $k_{\|} / k_{\perp}$ and moves a LH plasmon toward a dispersion less condition $\omega=\omega_{\mathrm{LH}}$ at which point the scattering ceases because frequencies below $\omega_{\mathrm{LH}}$ does not exist for the intermediate frequency waves in the electrostatic limit. This implies that within the electrostatic framework an ensemble of LH plasmons behaves as a "Bose gas," whose ultimate NL fate is to condense to the lowest energy state at $\omega=\omega_{\mathrm{LH}}$ and remain there as time stationary condensate. In a nonisothermal $\left(T_{e} \gg T_{i}\right)$ plasma the condensate may be unstable to modulation instability resulting in the formation of coherent structures, which may collapse and dissipate the energy as plasma heating. Langmuir wave evolution is an example of such behavior. ${ }^{9.15}$

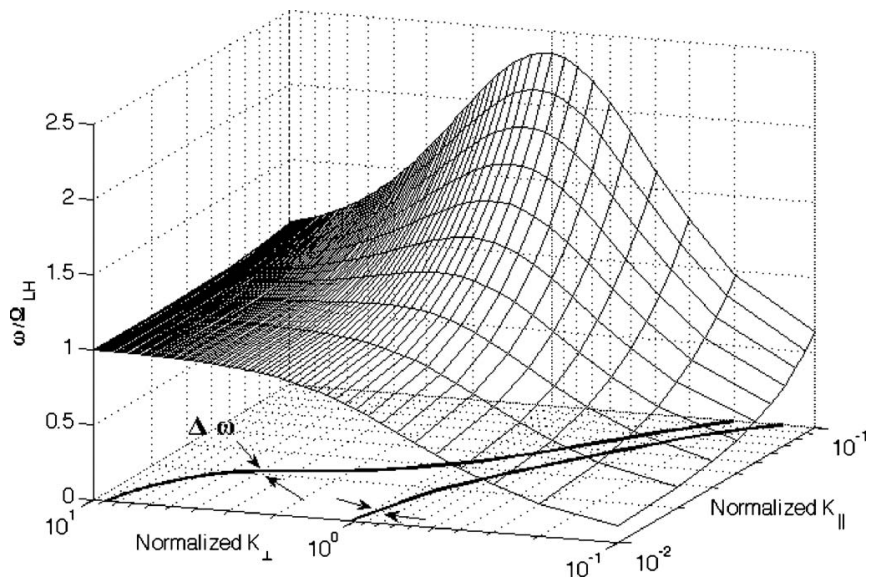

FIG. 1. The dispersion surface of the intermediate frequency electromagnetic whistler waves. The isofrequency lines indicate that there can be large change in the wave propagation angle due to NL scattering without much change in the frequency. Note that there is no minimum at $\omega=\omega_{\mathrm{LH}}$.

For narrow band LH waves isotropically distributed across magnetic field in the subsonic ion condition the real parts of Eqs. (4) and (7) lead to the nonlinear Schrodinger (NLS) equation. It has been suggested that LH coherent structures described by the solutions of the NLS equation may collapse and dissipate energy, which is of particular interest to the ionospheric plasmas. ${ }^{16}$ However, for waves in an incoherent broadband spectrum, which is usually the case in space plasmas, it is difficult to maintain the phase coherence necessary for the NLS equation solutions because of competition from induced wave-particle scattering. The induced scattering rate is comparable to the rate of creation and collapse of LH coherent structure. Scattering may remove the waves from the density cavity that traps the LH waves and thereby impede in the formation of coherent structures. This important wave loss mechanism is not included in a fluid theory based analysis of the phenomenon. ${ }^{8,17}$ Perhaps due to these impediments to the formation of coherent structures unambiguous evidence of the LH collapse phenomenon in the ionosphere is absent. ${ }^{18}$ Hence, we seek an alternate and credible description for the evolution of LH turbulence.

As remarked earlier, the LH waves are not purely electrostatic and hence it is inappropriate to treat their NL evolution within an electrostatic framework. To address this problem we must generalize the Hasegawa and $\mathrm{Chen}^{14}$ formalism to the electromagnetic regime. Electromagnetic generalization of Eq. (10) is

$$
\begin{aligned}
\gamma_{\mathrm{NL}} \sim & \frac{\omega_{p e}^{2}}{\omega_{k 2}} \frac{\vec{k}_{2}^{2}}{1+\vec{k}_{2}^{2}} \sum_{k 1} \frac{\left(\vec{k}_{1} \times \vec{k}_{2}\right)_{\|}^{2}}{k_{1 \perp}^{2} k_{2 \perp}^{2}} \frac{\vec{k}_{1}^{2}}{1+\vec{k}_{1}^{2}} \\
& \times \frac{\pi \zeta \operatorname{Im} Z(\zeta)}{\left[1+\left(\omega_{k 1}-\omega_{k 2}\right)^{2} /\left(\vec{k}_{1}-\vec{k}_{2}\right)^{2} c_{s}^{2}\right]^{2}} \frac{\left|E_{k 1}\right|^{2}}{4 \pi n_{0} T_{e}} .
\end{aligned}
$$

Figure 1 is the electromagnetic dispersion plot [Eq. (3)] for the intermediate frequency waves. The electromagnetic dispersion character is significantly different from that obtained from the electrostatic approach, $\omega=\omega_{\mathrm{LH}}\left(1+k_{\|}^{2} m_{i} / k_{\perp}^{2} m_{e}\right)^{1 / 2}$, which indicates a minimum at $\omega=\omega_{\mathrm{LH}}$. Now there is no minimum at $\omega=\omega_{\mathrm{LH}}$ and hence the NL evolution does not 
lead to the formation of condensates. Isofrequency lines in Fig. 1 indicate a variety of paths through which the mother wave can be scattered into a daughter wave with large change in $|k|$ and in the propagation angle $k_{\|} / k_{\perp}$ but with small change in frequency, $\left(\omega_{k 1}-\omega_{k 2}\right)<\left|\vec{k}_{1}-\vec{k}_{2}\right| c_{s}$. This is similar to the diffraction phenomenon. The density irregularity, $\delta n_{e}^{(2)}$ [Eq. (7)], acts as an efficient diffraction grating with slit width equal to the wavelength of the beat wave.

For a broadband turbulence $\left(\delta \omega \gg \bar{k}_{1} c_{s}\right)$ the rate for a mother wave $\left(\vec{k}_{1}, \omega_{k 1}\right)$ to scatter into a daughter wave $\left(\vec{k}_{2}, \omega_{k 2}\right)$ is obtained by adding the contributions from all possible mother waves within the frequency interval $\omega_{k 2}+\Delta \omega$, where $\Delta \omega \sim\left|\vec{k}_{1}-\vec{k}_{2}\right| c_{s} \sim\left(\bar{k}_{1}+\bar{k}_{2}\right) \beta_{e}^{1 / 2} \omega_{\mathrm{LH}}$. This leads to an estimate for the rate

$$
\gamma_{\mathrm{NL}} \sim \frac{\omega_{p e}^{2}}{\delta \omega} \frac{\omega_{\mathrm{LH}}}{\omega}\left(\frac{1}{\beta_{e}}\right)^{1 / 2} \frac{\vec{k}_{2}^{2}}{1+\vec{k}_{2}^{2}} \sum_{k_{1}} \frac{E_{k 1}^{2}}{B_{0}^{2}} \frac{\vec{k}_{1}^{2}\left(\bar{k}_{1}+\bar{k}_{2}\right)}{1+\vec{k}_{1}^{2}} .
$$

Here the summation is with $\vec{k}_{1}$ along the characteristic $\omega\left(\vec{k}_{1}\right)=\omega\left(\vec{k}_{2}\right)$.

Since $\gamma_{\mathrm{NL}} \propto\left(\vec{k}_{1} \times \vec{k}_{2}\right) \cdot \vec{b}_{0}$ [Eq. (11)], large angle scattering is more likely. Consequently, quasielectrostatic waves scatter into electromagnetic waves. Electromagnetic waves have large group velocity, which allows the energy to convect out of the region of creation. While the wave vector spectrum changes substantially due to repeated scattering the frequency spectrum remains relatively unaffected. As a result, it is not possible to glean the trajectory of the energy flux in the whistler turbulence from the wave vector spectrum. Therefore, in whistler turbulence the wave vector spectrum is not a surrogate for the frequency spectrum. A steady state may be achieved by a balance between the pump and convection of energy. Redistribution of energy globally in the physical space rather than local dissipation emerges as the NL end stage of the LH turbulence instead of condensation or creation and collapse of coherent structures. Most of the energy may convect away instead of being dissipated locally. In contrast, however, for the purely electrostatic Langmuir waves with small group velocity, there is good experimental evidence for condensation and collapse. ${ }^{19,20}$ Hence, as remarked earlier, the formally electromagnetic nature of the $\mathrm{LH}$ waves leads to a significantly different saturation mechanism for the LH turbulence.

\section{TWO-DIMENSIONAL ELECTROMAGNETIC PIC SIMULATION}

For self-consistent numerical assessment of the above it is necessary to include the new term proportional to $\delta n$, ideally in a three-dimensional electromagnetic PIC simulation. However such simulation is still impractical, therefore we use a two-dimensional (2D) $(x, y)$ electromagnetic PIC model but with $B_{0}$ in the $(x, z)$ plane at an angle $\theta$ such that $\theta=0$ implies that $B_{0}$ is directed along the $x$ axis. This activates the $(\vec{E} \times \vec{\nabla} \delta n) \cdot \vec{b}_{0}$ term in Eq. (4) that would be lost if $B_{0}$ is in the simulation $(x, y)$ plane. If $\theta=90^{\circ}$ and $k_{\|}=k_{x} \cos \theta=0$ then this important term would also be lost because there will be no $\delta n$ due to pondermotive force along $B_{0}$. Thus, it is possible to demonstrate the crucial 3D NL

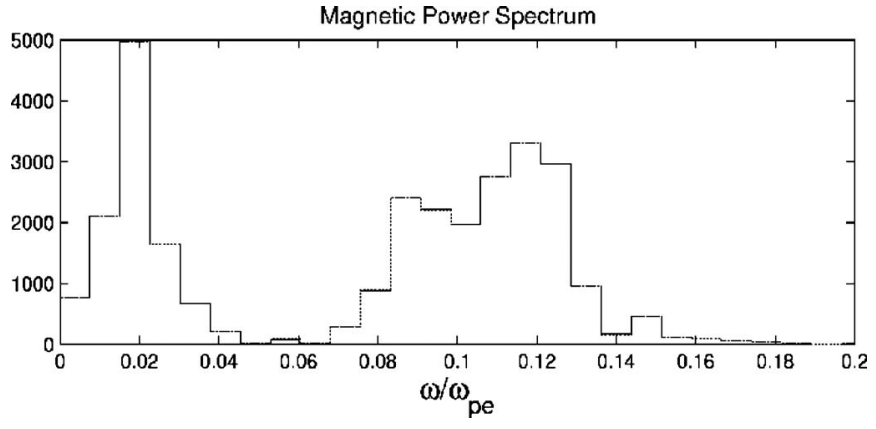

FIG. 2. Frequency spectrum at $\omega_{p e} t=830$ of wave magnetic field for whistler waves for $B_{0}$ at $\theta=60^{\circ}$ and $m_{r}=3 m_{e}$. The initial whistler decays into a daughter whistler by radiating a lower frequency MS/LH wave.

effect in a relatively simple 2D PIC simulation by choosing $0<\theta<90^{\circ}$. Even with this simplification it is not trivial to simulate a fully developed whistler turbulence involving many waves. We therefore focus on the properties of NL scatterings which is the essential ingredient that characterizes whistler turbulence. By appropriately choosing parameters we can study the roles of both the wave-wave and waveparticle scatterings arising due to the vector nonlinearity in the simulation. In general, dimension of the simulation box and symmetrical boundary condition restrict the wave spectrum generated because of quantization of the waves. Because of this limitation three wave resonance conditions, i.e., $\omega_{k 1}=\omega_{k 2}+\omega_{k 1-k 2}, \vec{k}_{1}=\vec{k}_{2}+\vec{k}_{3}$, are more difficult to satisfy in the simulation box than the Landau resonance with electrons, i.e., $\left(\omega_{k 1}-\omega_{k 2}\right)-\left(k_{1 \|}-k_{2 \|}\right) \mathrm{v}_{t, \|}=0$.

The simulation model is described in detail by Wang et $a .^{21}$ We choose a simulation plane $(x, y)$ with dimension $(512 \times 256) \lambda_{\mathrm{De}}$, where $\lambda_{\mathrm{De}}$ is the electron Debye length. This is equivalent to $(51.2 \times 25.6) c / \omega_{p e}$. The cell size $\lambda_{\mathrm{De}}=0.1 \mathrm{c} / \omega_{p e}$ and we use 81 particles/cell. The parameters chosen are $m_{i} / m_{e}=100, \quad \omega_{p e} / \Omega_{e}=\sqrt{5}, \quad \mathrm{v}_{t e}=0.14 c$, $\beta_{e} \equiv n T_{e} /\left(B_{0}^{2} / 8 \pi\right)=0.1$, and $T_{e}=T_{i}$. The waves are initiated in the system self-consistently by a perpendicular ring distribution. We choose "heavy electrons" as the ring species with mass $m_{r}=(3$ and 10$) m_{e}$, ring velocity of $V_{r}=0.2 c$, and a ring density of $n_{r}=0.25 n_{e}$. A ring distribution can trigger both oblique and parallel propagating whistlers. The highly oblique whistlers are triggered at ring cyclotron harmonics in a manner similar to that described by Ganguli et $a .^{22}$ for the lower frequency shear Alfven waves, while the parallel whistlers are triggered due the temperature anisotropy ${ }^{23}$ introduced by the ring distribution.

Figure 2 is the frequency spectrum for waves when $\theta=60^{\circ}$ and $m_{r}=3 m_{e}$. Initially whistler waves in this parameter regime is generated for $\omega=0.15 \omega_{p e} \sim 3.3 \omega_{\mathrm{LH}}$. Linear analysis for the simulation parameters shows that oblique whistlers with large $k_{\perp}$ can be triggered self-consistently by the ring distribution at first harmonic of the ring cyclotron frequency. At $\omega_{p e} t=830$ the frequency spectrum shows the mother whistler decaying into a daughter whistler at $\omega \sim 0.1 \omega_{p e}$ along with the radiation of a lower frequency $\omega \sim 0.5 \omega_{\mathrm{LH}} \mathrm{MS} / \mathrm{LH}$ wave. Figure 3(a) and 3(b) are the $k$ spectra for the wave electric fields. At $\omega_{p e} t=470$ the initial 

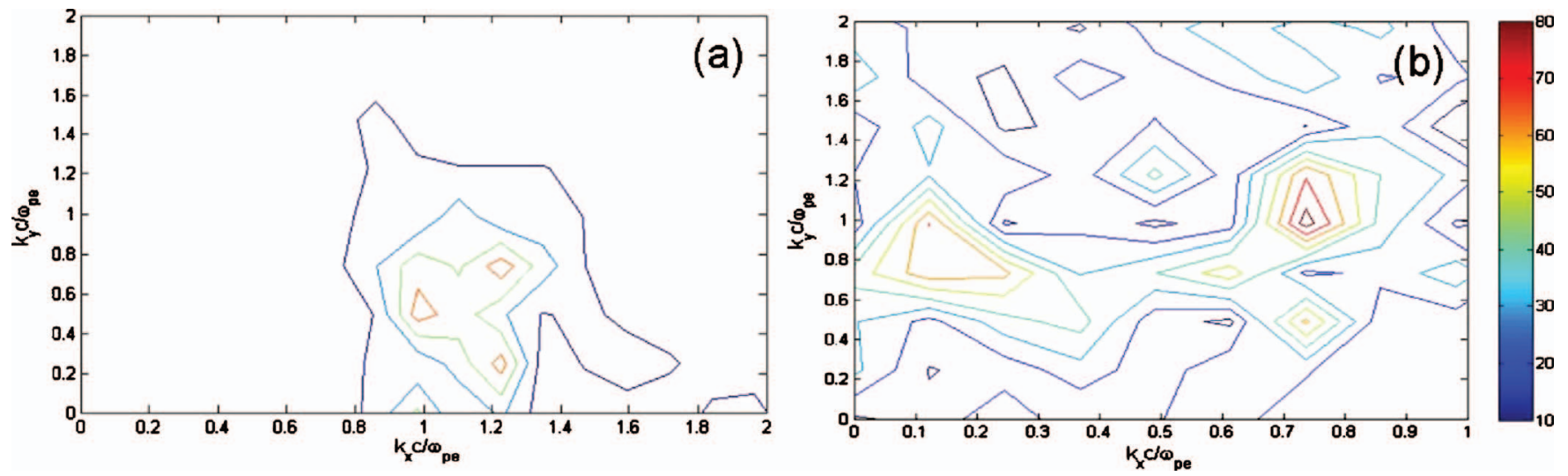

FIG. 3. (Color) (a) The wave vector spectrum corresponding to Fig. 2 for the initial whistler electric field at $\omega_{p e} t=470$ and (b) same figure as in (a) but at a later time at $\omega_{p e} t=750$ that shows a well developed daughter whistler and the lower frequency wave.

wave is generated for $\bar{k}_{x} \sim 1.3, \bar{k}_{y} \sim 0.8$, and $\bar{k}_{\|}=\bar{k}_{x} / 2 \sim 0.65$. At a later time, $\omega_{p e} t=750$, signatures of a daughter whistler at $\bar{k}_{x} \sim 0.75, \bar{k}_{y} \sim 1$, and $\bar{k}_{\|}=\bar{k}_{x} / 2 \sim 0.38$ and a lower frequency MS wave at $\bar{k}_{x} \sim 0.15, \bar{k}_{y} \sim 0.9$, and $\bar{k}_{\|}=\bar{k}_{x} / 2 \sim 0.07$ are visible. This is an example of NL W/MS/LH wave-wave scattering through parametric decay process in the simulation. However, wave-particle scattering is also prevalent in the simulation.

Evidence for wave-particle scattering becomes clearer for $m_{r}=10 m_{e}$ and $\theta=78^{\circ}$. Figures 4(a)-4(d) show the frequency spectra at four different time intervals. Initial whistler wave in this parameter regime is generated as in the previous case at the first harmonic of the ring cyclotron frequency but with $\omega=0.044 \omega_{p e} \sim \omega_{\text {LH }}$. Figures $4(\mathrm{a})-4(\mathrm{~d})$ show generation of the initial whistler and then subsequent multiple scatterings from plasma particles into daughter whistlers. Absence of a low frequency satellite, which was present in the previous case, is a confirmation of wave-particle scattering. Inspection of Fig. 4(d) indicates that $\left(\omega_{k 1}-\omega_{k 2}\right) \sim 0.005 \omega_{p e} \sim 0.1 \omega_{\mathrm{LH}}$ while $\left|\vec{k}_{1}-\vec{k}_{2}\right| c_{s}$ $\sim \bar{k}_{1}\left(\beta_{e}\right)^{1 / 2} \omega_{\mathrm{LH}} \sim 0.15 \omega_{\mathrm{LH}}$. Thus, the subsonic ion condition $\left(\omega_{k 1}-\omega_{k 2}\right)<\left|\vec{k}_{1}-\vec{k}_{2}\right| c_{s}$ is satisfied. Also $\left(k_{1 \|}-k_{2 \|}\right) \mathrm{v}_{t e}$ $=\Delta \bar{k}_{x} \cos \theta\left(m_{i} / m_{e}\right)^{1 / 2} \beta^{1 / 2} \omega_{\mathrm{LH}} \sim 0.25 \omega_{\mathrm{LH}}$ is of the same order as $\left(\omega_{k 1}-\omega_{k 2}\right)$ and hence the Landau resonance condition $\left[\left(\omega_{k 1}-\omega_{k 2}\right) \approx\left(k_{1 \|}-k_{2 \|}\right) \mathrm{v}_{t e}\right]$ is also satisfied. In addition, Figs. 5(a) and 5(b) indicate large angle scattering in the simulation as expected. This shows that NL scattering alters the wave vector spectrum significantly but the change in the frequency spectrum is mild.

To unambiguously establish the dominance of the vector nonlinearity in determining the course of evolution of the whistler turbulence, we eliminate the term proportional to $\delta n$
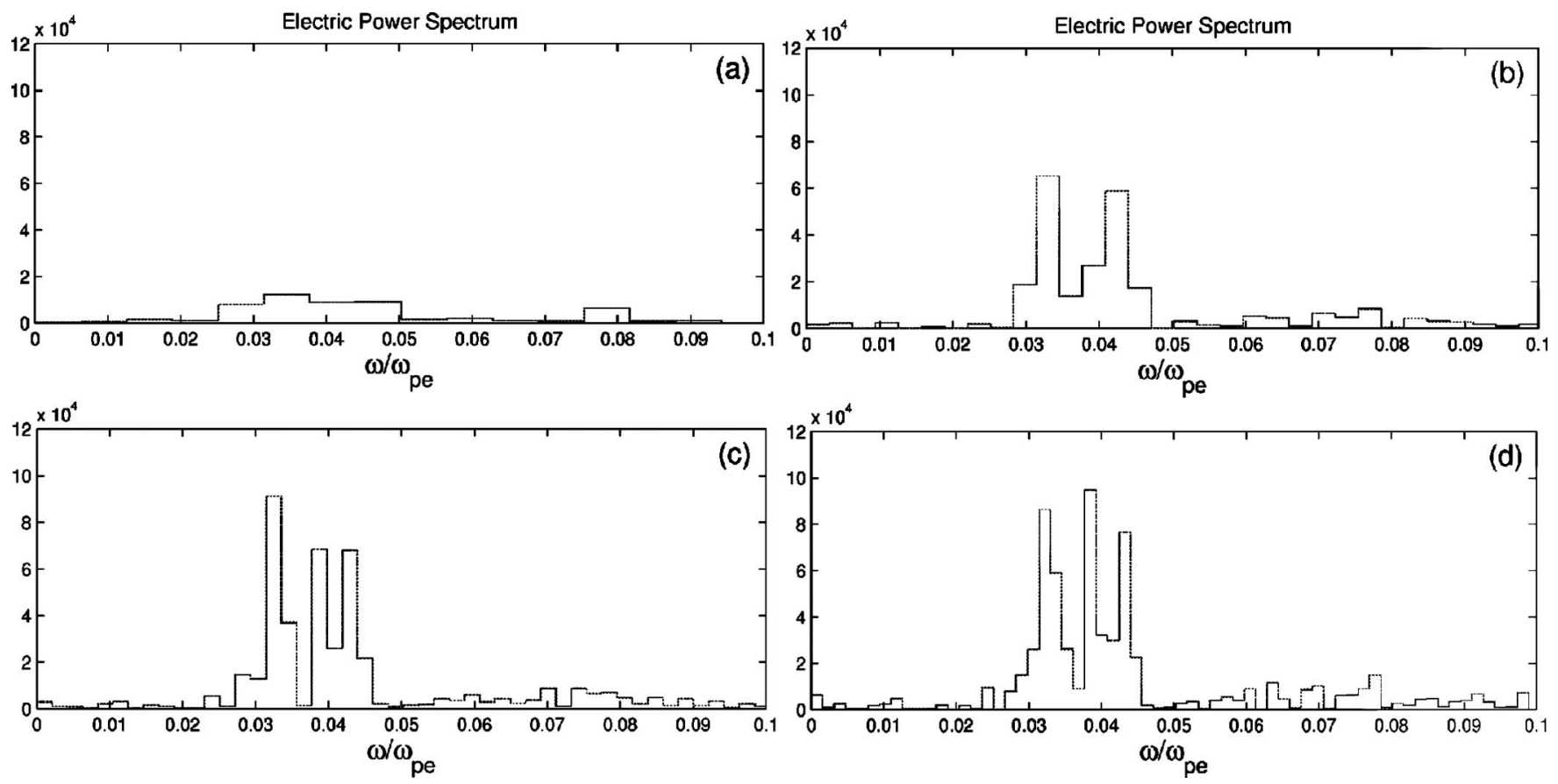

FIG. 4. Frequency spectra for time intervals (a) $0<\omega_{p e} t<1000$, (b) $0<\omega_{p e} t<2000$, (c) $0<\omega_{p e} t<3000$, and (d) $0<\omega_{p e} t<4000$ of the wave electric field for whistler waves for $\theta=78^{\circ}$ and $m_{r}=10 m_{e}$. The initial whistler decays into a daughter whistler through the Landau resonance with the thermal electrons. 

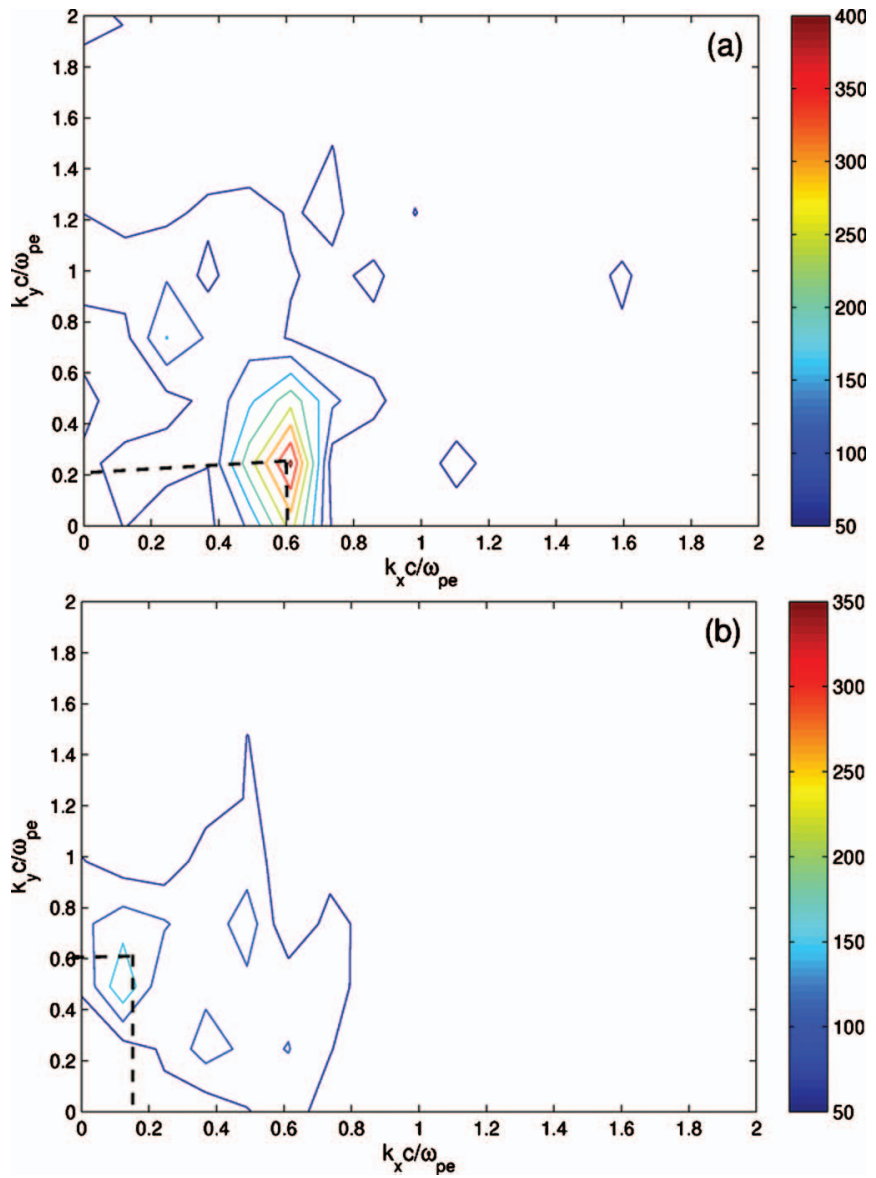

FIG. 5. (Color) The wave vector spectrum for the waves described in Fig. 4 at (a) $\omega_{p e} t=1000$ and (b) $\omega_{p e} t=2000$. The mother wave (a) experiences a large angle scattering (b).

[in Eq. (4)] from the simulation model by making $\theta=0^{\circ}$ so that $B_{0}$ is in the $x$ direction in the simulation plane. This configuration is identical to that of Saito et al. ${ }^{5}$ In this case the strong temperature anisotropy of ring distribution species drives whistlers, as shown in Figs. 6(a) and 6(b). Figures 6(a), 6(b), 7(a), and 7(b) indicate that the initial whistler grows in amplitude with time but without the signatures of NL scatterings even at $\omega_{p e} t=5000$, which is much longer than the previous cases with tilted $B_{0}\left(\theta \neq 0^{\circ}\right)$ that included the vector nonlinearity and showed evidence of scattering at $\omega_{p e} t=750$ (Figs. 2 and 3) or at $\omega_{p e} t=2000$ (Figs. 4 and 5).
This leads to the conclusion that the vector nonlinearity introduced through the term proportional to $\delta n$ [in Eq. (4)] dominates the physics of whistler turbulence and determines its character. This dominant physics is lost in a 2D PIC simulation if $B_{0}$ is maintained in the simulation plane or in a 3D fluid simulation, which is based on an incompressible fluid model. Even in a 3D compressible fluid simulation contribution from the wave-particle scattering will be lost.

It should be noted however that if the $\theta=0^{\circ}$ simulation was run longer, then the scalar nonlinearity would have eventually introduced NL effects. The general expression for NL scattering by electrons that includes both vector and scalar nonlinearities is estimated as

$$
\begin{aligned}
\gamma_{\mathrm{NL}} \sim & \sum_{k 1}\left\{\frac{\Omega_{e}^{2}}{\omega} \frac{\vec{k}_{2}^{2}}{1+\vec{k}_{2}^{2}} \frac{\vec{k}_{1}^{2}}{1+\vec{k}_{1}^{2}} \frac{\left[\left(\vec{k}_{1} \times \vec{k}_{2}\right) \cdot \vec{b}\right]^{2}}{k_{1 \perp}^{2} k_{2 \perp}^{2}}+\frac{n_{0} \partial \omega}{\partial n_{0}}\right\} \\
& \times \frac{\zeta_{e} \operatorname{Im} Z\left(\zeta_{e}\right)}{\left[1+\left(\omega_{k 1}-\omega_{k 2}\right)^{2} /\left(\vec{k}_{1}-\vec{k}_{2}\right)^{2} c_{s}^{2}\right]^{2}} \frac{\omega_{p e}^{2}}{\Omega_{e}^{2}} \frac{\left|E_{k 1}\right|^{2}}{4 \pi n_{0} T_{e}} .
\end{aligned}
$$

The first and second terms in the curly bracket represent the vector and scalar nonlinearities, respectively. The scalar nonlinearity is smaller than the vector nonlinearity by a factor that scales as $\left(\omega / \Omega_{e}\right)^{2}$.

We also point out that in a broad frequency bandwidth turbulence the NL scattering rate $\gamma_{\mathrm{NL}} \propto 1 / \beta_{e}^{1 / 2}$, as long as $\beta_{e}>\left(m_{e} / m_{i}\right) /\left(\bar{k}_{1}+\bar{k}_{2}\right)^{2}$, which is necessary for maintaining the condition of unmagnetized and subsonic ion motion in beat waves. Thus, the NL $\mathrm{LH} \rightarrow \mathrm{W}$ scatterings are expected to be intense in the low $\beta_{e}$ near-earth plasma environment. For narrow frequency bandwidth waves, parametric decay in isothermal plasma is possible through the generation of low frequency MS/LH waves. The rate for this process is $\gamma_{\mathrm{NL}} \propto \omega\left(E_{k 1} / B_{0}\right)$ (Ref. 24) and is independent of $\beta_{e}$. However, for the broadband case Eq. (9) indicates that the decay rate through the generation of MS/LH waves is $\gamma_{\mathrm{NL}} \propto \omega\left(E_{k 1} / B_{0}\right)^{2}$, which is smaller by a factor $\beta_{e}^{1 / 2}$ than the competing rate of scattering through resonance with thermal particles in a $\beta_{e}<1$ plasma [Eq. (12)].
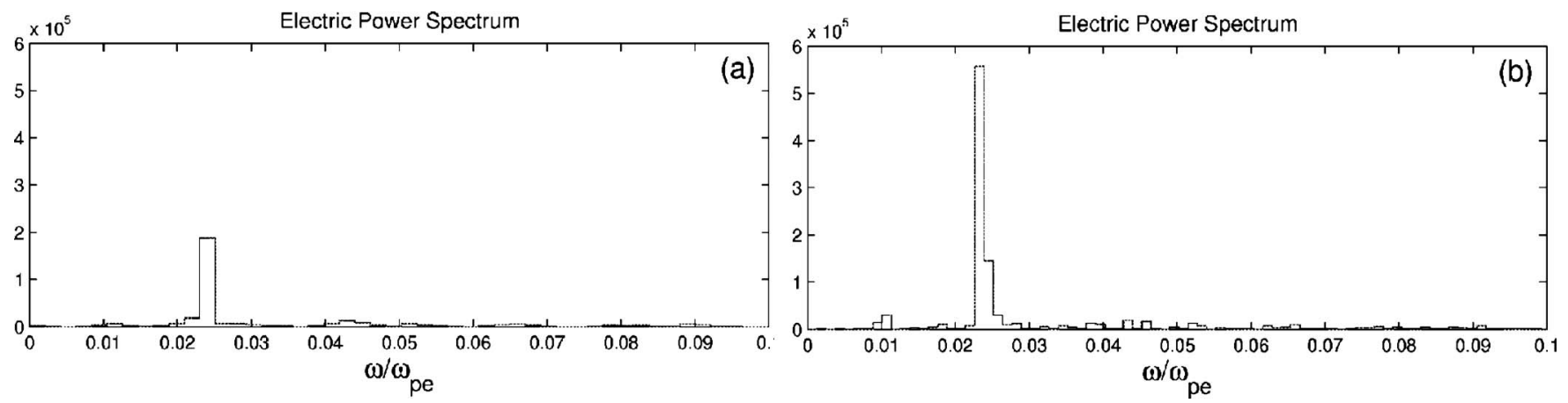

FIG. 6. Frequency spectra for time intervals, (a) $0<\omega_{p e} t<3000$ and (b) $0<\omega_{p e} t<5000$, of the wave electric field for whistler waves for $\theta=0^{\circ}$ and $m_{r}=10 m_{e}$. The initial whistler wave amplitude grows in time but signatures of NL scattering are not there. 

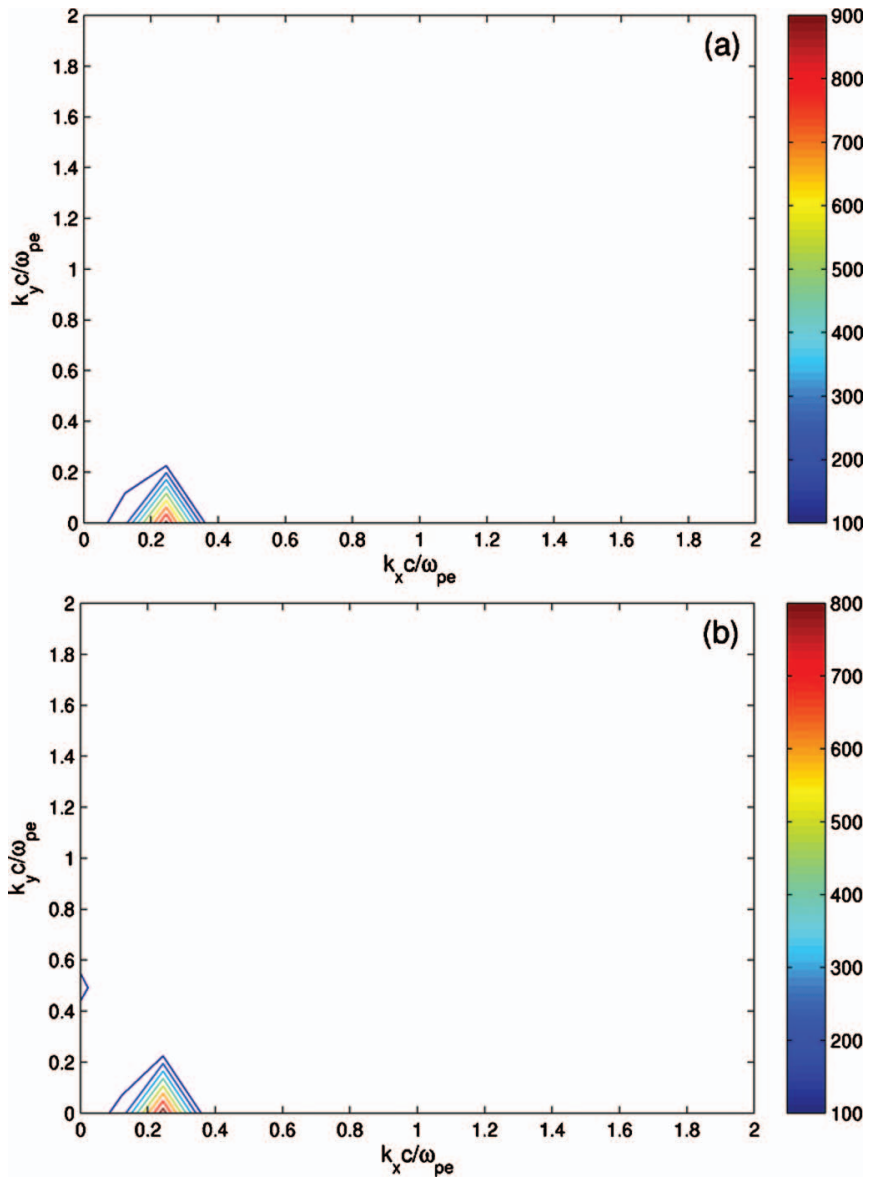

FIG. 7. (Color) Wave vector spectra corresponding to Fig. 6 at (a) $\omega_{p e} t=2000$ and (b) $\omega_{p e} t=5000$ of the wave electric field for $\theta=0^{\circ}$ and $m_{r}=10 m_{e}$. As in Fig. 6 there is no evidence of NL scattering.

\section{CONCLUSIONS}

We have analyzed the nature of electromagnetic turbulence in the intermediate frequency range $\left(\Omega_{i} \ll \omega \ll \Omega_{e}\right)$ in a low $\beta$ and isothermal plasma, which is typical in the nearearth space environment. We find that the vector nonlinearity dominates and gives rise to slow electron density irregularity due to the pondermotive force along the magnetic field, which induces strong NL scattering that changes the propagation angles of the waves substantially. Consequently, wave scattering converts quasielectrostatic waves into electromagnetic waves and vice versa, and thereby determines the character of the turbulence. Conversion of quasielectrostatic waves into electromagnetic and vice versa from a preexisting density structure is well known. ${ }^{25-27}$

NL wave coalescence and decay in homogeneous weak turbulence leads to cascade and energy flux toward higher frequencies. NL scattering on the other hand leads to inverse cascade toward lower frequencies. It is possible that in a certain range of plasma parameters and intermediate frequency range the wave-particle scattering primarily determines the evolution of the turbulence and the fate of the energy flux. As discussed earlier, the wave-particle scattering can convert quasielectrostatic waves with low group velocity into electromagnetic waves with large group velocity, which can convect the energy away from the region. This represents a possibility of wave energy loss without significant local plasma heating and may have applications in both terrestrial and extra-terrestrial plasmas. This is fundamentally different from neutral turbulence where forward cascade implies energy flux from long to short wavelengths until dissipation and the wave vector spectrum is a good surrogate for the frequency spectrum.

Typically whistler turbulence has been analyzed in a 2D simulation with the ambient magnetic field in the simulation plane. We established that the development of whistler turbulence is essentially a $3 \mathrm{D}$ phenomenon and the dominant vector nonlinearity is lost in a $2 \mathrm{D}$ simulation if the ambient magnetic field is maintained in the simulation plane. To demonstrate this we first used a 2D $(x, y)$ PIC simulation in which the consequences of the crucial 3D NL effects were simulated by maintaining the ambient magnetic field in the $(x, z)$ plane. Subsequently we repeated the same simulation with the ambient magnetic field in the simulation $(x, y)$ plane and found that the vector nonlinearity related effects disappeared. Because of the practical constraints a comprehensive 3D electromagnetic PIC simulation of the intermediate frequency turbulence remains beyond the scope of this article. However, the essential physics of induced NL scattering that dominates the dynamics and determines the character of the turbulence could be studied in sufficient detail using our simple 2D simulation model.

\section{ACKNOWLEDGMENTS}

This work was supported by the ONR and the DARPA. Stimulating discussions with Dennis Papadopoulos, Valentin Shevchenko, Parvez Guzdar, and Dan Winske are acknowledged. The views, opinions, and/or findings contained in this article/presentation are those of the author/presenter and should not be interpreted as representing the official views or policies, either expressed or implied, of the Defense Advanced Research Projects Agency or the Department of Defense.

${ }^{1}$ Y. Omura, D. Nunn, H. Matsumoto, and M. J. Rycroft, J. Atmos. Terr. Phys. 53, 351 (1991).

${ }^{2}$ R. A. Helliwell, Rev. Geophys. 26, 551 (1988).

${ }^{3}$ C. Cattell, J. R. Wygant, K. Goertz, K. Kersten, P. J. Kellogg, T. von Rosenvinge, S. D. Bale, I. Roth, M. Temerin, M. K. Hudson, R. A. Mewaldt, M. Wiedenbeck, M. Maksimovic, R. Ergun, M. Acuna, and C. T. Russell, Geophys. Res. Lett. 35, L01105, doi:10.1029/2007GL032009 (2008).

${ }^{4}$ E. M. Dubinin, M. Maksimovic, N. Cornilleau-Wehrlin, D. Fontaine, P. Travincek, A. Mangeney, O. Alexandrova, K. Sauer, M. Fraenz, I. Dondouras, E. Lucek, A. Fazakerley, A. Balough, and M. Andre, Ann. Geophys. 25, 303 (2007).

${ }^{5}$ S. Saito, S. P. Gary, H. Li, and Y. Narita, Phys. Plasmas 15, 102305 (2008).

${ }^{6}$ R. L. Stenzel, J. Geophys. Res. 104, 14379, doi:10.1029/1998JA900120 (1999).

${ }^{7}$ H. Ji, S. Terry, M. Yamada, R. Kulsrud, A. Kuritsyn, and Y. Ren, Phys. Rev. Lett. 92, 115001 (2004).

${ }^{8}$ V. D. Shapiro, V. I. Shevchenko, A. S. Sharma, K. Papadopoulos, R. Z. Sagdeev, and V. B. Lebedev, J. Geophys. Res. 98, 1325, doi:10.1029/ 92JA01729 (1993).

${ }^{9}$ V. E. Zakharov, Sov. Phys. JETP 35, 908 (1972). 
${ }^{10}$ V. M. Dikasov, L. I. Rudakov, and D. D. Ryutov, Sov. Phys. JETP 21, 608 (1965).

${ }^{11}$ K. Akimoto, K. Papadopolous, and D. Winske, J. Plasma Phys. 34, 445 (1985).

${ }^{12}$ M. Porkolab, S. Bernabei, W. M. Hooke, R. W. Moteley, and T. Nagashima, Phys. Rev. Lett. 38, 230 (1977).

${ }^{13}$ V. K. Tripathi, C. Grebogi, and C. S. Liu, Phys. Fluids 20, 1525 (1977).

${ }^{14}$ A. Hasegawa and L. Chen, Phys. Fluids 18, 1321 (1975).

${ }^{15}$ L. M. Degtyarev, V. E. Zakharov, and L. I. Rudakov, Sov. J. Plasma Phys. 2, 240 (1977).

${ }^{16}$ P. A. Robinson, A. Melatos, and W. Rozmus, Phys. Plasmas 3, 133 (1996).

${ }^{17}$ P. A. Robinson, Adv. Space Res. 23, 1679 (1999).

${ }^{18}$ P. W. Schuck, G. I. Ganguli, and P. M. Kintner, Phys. Rev. Lett. 89, 065002 (2002).
${ }^{19}$ A. Y. Wong and P. Y. Cheung, Phys. Rev. Lett. 52, 1222 (1984).

${ }^{20}$ W. Birkmayer, T. Hagfors, and W. Kofman, Phys. Rev. Lett. 57, 1008 (1986).

${ }^{21}$ J. Wang, P. Liewer, and V. Decyk, Comput. Phys. Commun. 87, 35 (1995).

${ }^{22}$ G. Ganguli, L. Rudakov, M. Mithaiwala, and K. Papadopolous, J. Geophys. Res. 112, A06231, doi:10.1029/2006JA012162 (2007).

${ }^{23}$ C. F. Kennel and H. E. Petschek, J. Geophys. Res. 71, 1 (1966).

${ }^{24}$ P. Kumar and V. K. Tripathi, Phys. Plasmas 15, 052107 (2008).

${ }^{25}$ T. F. Bell and H. D. Ngo, J. Geophys. Res. 95, 149, doi:10.1029/ JA095iA01p00149 (1990).

${ }^{26}$ B. Eliasson and K. Papadopolous, J. Geophys. Res. 113, A09315, doi:10.1029/2008JA013261 (2008).

${ }^{27}$ P. W. Schuck, J. W. Bonnell, and P. M. Kintner, Jr., IEEE Trans. Plasma Sci. 31, 1125 (2003). 